\title{
Historia digital y la cuestión de Palestina. Construyendo herramientas para combatir el memoricidio
}

\author{
Digital History and the Question of Palestine: \\ Developing Tools to Fight Memoricide
}

\author{
Diego Checa Hidalgo* \\ Universidad de Granada \\ https:/ / orcid.org/0000-0002-3269-0240 \\ diegoch@ugr.es \\ José Carvajal Martínez \\ Universidad de Granada \\ https:/ / orcid.org/0000-0003-0180-4134 \\ jcarmar26@gmail.com \\ Belén Habboob Martos \\ Universidad de Granada \\ https://orcid.org/0000-0002-8658-8807 \\ be195@correo.ugr.es
}

Recibido: 20/07/2021; Revisado: 13/12/2021; Aceptado: 29/12/2021

\section{Resumen}

Este artículo analiza algunas de las contribuciones que la historia digital está realizando en la preservación de la memoria histórica y en la construcción de narrativas alternativas que cuestionan los discursos tradicionales sobre la interpretación de los hechos del pasado en el caso de la cuestión palestina. Para ello, partiendo de un marco teórico ubicado en la historia y las humanidades digitales y presentando el contexto de la Nakba palestina y las políticas israelíes de memoricidio que sufre la población palestina, nuestra investigación estudia tres proyectos digitales significativos que pretenden recuperar la memoria de la Nakba y promover la elaboración de narrativas propias palestinas en torno a ella con notable éxito.

Palabras clave: Palestina, Israel, Nakba, memoria, humanidades digitales.

*Autor de correspondencia / Corresponding author.

Copyright: (C) 2022 ULPGC. Este es un artículo de acceso abierto distribuido bajo los términos de la licencia Creative Commons Atribución-NoComercial-SinDerivar (by-nc-nd) Spain 3.0. 


\begin{abstract}
This article analyses some of the main contributions of digital history in the preservation of historical memory and the construction of alternative narratives that dispute traditional discourses on the question of Palestine. This research is informed by digital history and the digital humanities within the context of the Nakba, as well as Israeli policies of memoricide against the Palestinian population. The article covers three significant digital projects that seek to recover the memory of the Nakba and cultivate Palestinian narratives around this phenomenon, with remarkable success.
\end{abstract}

Keywords: Palestine, Israel, Nakba, Memory, Digital Humanities.

\title{
1. INTRODUCCIÓN
}

Este artículo pretende participar en el debate sobre las contribuciones que puede realizar la historia digital a la construcción de un conocimiento alternativo que dispute las narrativas hegemónicas sobre la interpretación de los hechos del pasado y para la recuperación y preservación de la memoria histórica desde lógicas subalternas a partir del estudio del caso de la cuestión de Palestina. ${ }^{1}$

En 1948 la Nakba, resultado de una serie de dinámicas coloniales que culminaron en la expulsión de la mitad de la población autóctona de la Palestina histórica (BASALLOTE et al., 2017), supuso un suceso traumático que fue invisibilizado en la historiografía tradicional y desafió la propia existencia de la identidad palestina y la preservación de su memoria histórica. Identidad y memoria siguieron estando amenazadas por las políticas activas israelíes que prosiguieron la colonización del territorio, continuaron desplazando forzosamente a la población autóctona e imposibilitaron el retorno de las comunidades palestinas que habían buscado refugio en otros lugares huyendo de la limpieza étnica, en un proceso que el historiador israelí Ilan Pappé no dudó en denominar memoricidio (PAPPÉ, 2006).

Ante este contexto, la historia digital ofrece caminos para combatir el crimen del memoricidio y facilitar la creación de narrativas alternativas que visibilicen los procesos históricos que atraviesan las poblaciones subalternas. Desde su origen, le ha caracterizado su interés por la subalternidad, por sus amplios enfoques y por recurrir a fuentes que la historiografía tradicional no ha considerado de forma suficiente. Esto ha provocado una transformación de las metodologías y los relatos historiográficos. A su vez, la historia digital ha posibilitado un acercamiento diferente a temáticas como las relacionadas con Palestina-Israel. Así, ha permitido alejarse de los grandes discursos hegemónicos, basados en lógicas coloniales y neocoloniales, para construir un conocimiento histórico alternativo pero riguroso, cercano a las teorías decoloniales e incorporando saberes del sur global.

Precisamente, partiendo de un aparato conceptual elaborado a partir del marco de las humanidades digitales, este trabajo pretende analizar cómo la historia digital puede contribuir a la construcción de nuevos saberes y a la preservación de la memoria histórica en el caso de la población palestina cuya identidad se

1 Este artículo ha sido elaborado en el marco del proyecto «Fuentes digitales para la comprensión del mundo árabe: el caso de la cuestión palestina», financiado por el programa «Proyectos de Investigación del MediaLab» del Plan Propio de Investigación y Transferencia de la Universidad de Ganada correspondiente al año 2020. 
encuentra permanentemente amenazada por el poder colonial israelí, a partir del análisis de tres proyectos que abordan diferentes cuestiones relacionadas con la historia de la Nakba: Palestinian Oral History Archive, Zochrot Nakba Map y Palestine Open Maps.

El artículo se estructura en torno a cuatro apartados, precedidos por esta introducción y seguidos de unas conclusiones. Así, mientras el primer apartado muestra el planteamiento de la metodología utilizada en la investigación, el segundo apartado recoge el marco teórico y conceptual en el que se ha apoyado la investigación y que se ubica en el giro digital en la historia y las aportaciones de las humanidades digitales a la investigación. En tercer lugar, se presenta el contexto la Nakba y el memoricio del pueblo palestino para, en cuarto lugar, analizar los tres proyectos que contribuyen al estudio de la cuestión Palestina desde la historia digital y presentar, finalmente, los resultados alcanzados.

\section{METODOLOGÍA}

La metodología utilizada para desarrollar esta investigación ha consistido en un análisis documental de fuentes primarias y secundarias, que ha incluido una abundante bibliografía referente tanto a la temática relacionada con las humanidades y la historia digital, como a la cuestión de Palestina y la Nakba.

La búsqueda de proyectos para la recuperación de la memoria histórica palestina se ha realizado a partir de la revisión de la literatura existente y del rastreo de las iniciativas de la sociedad civil que han puesto en marcha acciones digitales en este sentido. Este proceso implicó una selección de tres proyectos con la intención de obtener una muestra representativa, variada y que permitiese explorar los objetivos de este trabajo. De los proyectos identificados, se han seleccionado tres que son significativos, tienen una trayectoria de trabajo notable, ofrecen diferentes metodologías y herramientas para abordar su propósito y disponen de plataformas digitales con bases de datos abiertas que permiten acceder fácilmente a sus recursos. Además de los proyectos seleccionados para su análisis, durante esta investigación se han explorado otras que finalmente no se han incluido en este trabajo por ser poco relevantes, tener un alcance limitado en sus objetivos o no disponer de una trayectoria suficientemente afianzada.

Una vez seleccionados los tres proyectos a estudiar, (Palestinian Oral History Archive, Zochrot Nakba Map y Palestine Open Maps), se analizaron sus orígenes, sus propósitos y su alcance, se identificaron sus metodologías y se valoraron sus resultados y sus contribuciones en torno a dos cuestiones: la preservación de la memoria histórica Palestina y la generación de narrativas propias de las comunidades que les permitan resistir las políticas que promueven el memoricidio.

El análisis de los recursos digitales desde una perspectiva educativa ha sido fundamental en la construcción metodológica de esta investigación. Si tenemos en cuenta que para este tipo de proyectos es tan importante la generación de conocimientos como su difusión hacia la sociedad, dónde identificar y entender los mecanismos de esta transferencia ha resultado fundamental para nuestro trabajo. De tal forma que los tres proyectos han sido estudiados en base a una perspectiva didáctica, teniendo en cuenta su potencial para la enseñanza de la historia y su capacidad de transferencia como señalan Willyan DA SiLva y Claudio ZARATE (2021). 


\section{EL GIRO DIGITAL Y LA HISTORIA}

El giro digital está cambiando el modo en el que los historiadores realizan sus investigaciones. El cambio no solamente está orientado por los procesos de digitalización de archivos que facilitan el acceso de los historiadores a las fuentes, sino que implica transformaciones más profundas que suponen el empleo de herramientas para consultar mayores bases de datos y la aplicación de nuevas metodologías para abordar las preguntas de investigación, está ampliando los horizontes de los investigadores (PONS, 2011; TER BRAAKE et al., 2016). Estas transformaciones han dado lugar a la aparición de la historia digital, que comparte con las humanidades digitales la complejidad del campo de estudio, la necesidad de enfoques interdisciplinares y el establecimiento de procesos cooperativos, transparentes y abiertos para la construcción del conocimiento.

Las humanidades digitales son un área de investigación en expansión cuyas aportaciones están contribuyendo a la renovación de las Ciencias Humanas desde hace ya más de cincuenta años. Tal y como señalaba el profesor Antonio Rojas Castro: «Más que una disciplina homogénea caracterizada por el uso de instrumentos digitales, las humanidades digitales pueden describirse como un conjunto de principios, valores y prácticas en donde convergen múltiples objetos de estudio y saberes cuyas fronteras se encuentran en continua negociación» (RojAs CASTRO, 2013a: 79). Así, humanidades digitales es un concepto que comprende investigaciones que participan de los objetos de estudio de las Ciencias Humanas y Sociales e incorpora unas metodologías relacionadas con los procesos de digitalización experimentados por nuestras sociedades y que modifican las condiciones de producción de los saberes (DAcos, 2011). Podemos decir que este término es una noción inclusiva que permite hablar de diferentes iniciativas y actividades situadas en la intersección entre las humanidades y las tecnologías de la información o lo digital (Svensson, 2010). Es de destacar el amplio abanico de perspectivas, análisis y objetos de estudio que ofrece, puesto que a la visión instrumental de la tecnología digital añaden lo digital como objeto de estudio.

Esta área de investigación presenta unos elementos propios que determinan la manera en la que se construye el conocimiento. Una de sus características es su gran complejidad y diversidad, ya que no es una disciplina unificada, sino que engloba una serie de prácticas convergentes cuya institucionalización se ha afianzado (SvENSson, 2010). Fruto de esta diversidad aparece otra de sus principales particularidades como es su naturaleza interdisciplinar, que obliga a los investigadores a colaborar con pares de disciplinas diferentes a las suyas (RojAS CASTRO, 2013a: 76-79). Otro de sus elementos relevantes deriva de los valores intrínsecos en las humanidades digitales y de las prácticas de sus investigadores, que presentan una fuerte conciencia sobre la producción y difusión del conocimiento de manera abierta y transparente, para facilitar la colaboración entre pares, pero también para democratizar el acceso a los saberes (Rojas CASTRO, 2013a: 89-92). En este sentido, las redes sociales también juegan un papel importante en las humanidades digitales porque suponen nuevos cauces de transferencia del conocimiento y fomentan la creación de vínculos comunitarios.

Como área de investigación, las humanidades digitales han evolucionado desde la década de 1960, pudiendo establecerse dos fases: Las Humanidades 1.0 y las Humanidades 2.0, utilizando la distinción entre Web 1.0 y Web 2.0, para señalar una transición de la computación en humanidades a las humanidades 
multimodales más interactivas, abiertas y participativas (SvENSSON, 2010; GALLINI y NoIRET, 2011). Con más recorrido histórico en el mundo anglosajón (HOCKEY, 2004; SCHREIBMAN, SiEMENS y UnSWORTH, 2004), este campo de estudio es reciente en el ámbito académico del mundo hispano, aunque su relevancia ha ido creciendo en la última década (ToscANo et al., 2020). La confluencia entre humanidades e informática en este ámbito se fue produciendo desde finales de la década de 1980 pero no fue hasta comienzos del s. XXI cuando se comenzó a dar difusión a este término en España y empezó su proceso de institucionalización que llevó al nacimiento de la asociación de Humanidades Digitales Hispánicas en 2011 (RojAs, 2013b: 10-11).

Evidentemente, tanto las humanidades como la historia digital son campos en los que convergen humanidades e historia con las nuevas tecnologías, la informática o el ámbito web (COHEN y RosenZWEIG, 2006). Una de las definiciones más acertadas y que mejor nos revela lo que supone la historia digital es la que se propuso hace más de una década en el «Interchange: The Promise of Digital History», en el marco del debate promovido por The Journal of American History (CoHEN et al., 2008: 454), que indica que «La historia digital puede ser entendida como una propuesta para el examen y la representación del pasado que trabaja con las nuevas tecnologías comunicativas del ordenador, de internet y de los sistemas de software». Estas palabras nos revelan cómo la historia necesariamente ha establecido unos vínculos vitales con las nuevas tecnologías, todos ellos con el objetivo de transformar unos aspectos metodológicos, teóricos y prácticos que han adaptado la disciplina histórica al siglo XXI.

Esto fue algo que se comprendió pronto en el ámbito de la historia contemporánea española. Así, en el primer congreso de la Asociación de Historia Contemporánea celebrado en 1992, el profesor Antonio Rodríguez de las Heras presentó una comunicación sobre «La integración de la informática en el trabajo del historiador». En 1996, este tema seguía siendo relevante y fue tratado en el tercer congreso de la asociación por el Profesor Miguel Artola en su ponencia sobre «Historiografía e informática» (PONS y EIROA, 2018: 14-15). Luego, estas cuestiones casi desaparecieron del radar de la historiografía española salvo impulsos esporádicos como, por ejemplo, los de Anaclet Pons (2011; 2013), hasta que en los congresos de 2014 y 2016 vuelven a aparecer con fuerza en talleres dedicados a ellas. De la creciente atención que le presta la historiografía española da cuenta el monográfico sobre historia digital que la revista Ayer publica en su volumen 110 número 2 de 2018, considerándola como «una apuesta del siglo XXI».

Está claro que la historia digital presenta un valor para las humanidades y la investigación histórica en general que va más allá del interés por digitalizar el pasado y ahora despliega un enfoque más aplicado y amplio que permite superar el debate que se planteaba el mundo académico acerca de sus aportes sobre el conocimiento del pasado, más allá de su representación de una forma y perspectiva diferentes (Pons, 2018: 41-42). De tal modo que a día de hoy podría decirse que la relevancia de la historia digital radica en que es una historia global que ha trascendido fronteras historiográficas o lingüísticas, fruto del contexto globalizado en el que se desarrolla (Melo, 2011: 84). Un carácter global que determina también otra de sus elementos más reconocibles, como es la necesidad de desarrollar marcos de trabajo colaborativos que permitan abordar investigaciones con ingentes cantidades de datos desde enfoques interdisciplinares. A su vez, conviene señalar que los proyectos y trabajos de historia digital pueden 
diferenciarse en dos grupos: aquellos que tienen carácter académico y los que tienen una vertiente más divulgativa. Es significativo que éstos últimos suelen tener una mayor repercusión social gracias a su interés pedagógico y al uso consciente y estratégico de las redes sociales como herramientas digitales para la construcción y difusión del conocimiento.

Es interesante comprobar cómo la historia digital presta una especial atención a la historia de las comunidades de base, a partir de la acumulación de historias locales, interesándose por los actores subalternos y las narrativas que emergen de ellos para enfrentar discursos hegemónicos en un contexto globalizado. Es por ello que algunos de los proyectos académicos de historia digital más importantes y de mayor relevancia se centran en la subalternidad, en sujetos e historias que la historiografía tradicional ha abandonado. Una serie de proyectos, trabajos o propuestas cuya importancia radica en el uso de la historia digital como forma de recuperación de toda aquella memoria colectiva que en los archivos tradicionales se puede recuperar de forma limitada. Así, la historia digital utiliza los recursos considerados como «digitales», fruto de una investigación previa o no (BOCANEGRA BARBECHO et al., 2021: 13), como podrían ser documentos originales digitalizados, prensa o fotografías de la época en formato digital o grabaciones de entrevistas. De tal modo que la memoria colectiva que se intenta conformar pasa a ser una «memoria digital».

Los proyectos de historia digital a nivel académico han sabido adaptarse con el paso de los años, mejorando el uso de los recursos tecnológicos y digitales. Algunos de los más destacados se centran en esa memoria colectiva subalterna, como es el caso de los proyectos que se analizan en esta investigación. Así, son investigaciones que contrastan con todas aquellas propias de la historiografía tradicional, conocidas por diseccionar grandes archivos o fuentes fruto de los relatos hegemónicos.

Más allá de los cambios que ha producido a nivel académico en las últimas dos décadas, la historia digital ha transformado por completo las formas y la realidad de la divulgación histórica. Se ha pasado de utilizar soportes físicos como libros y revistas a utilizar medios digitales como blogs, revistas y otros múltiples tipos de espacios virtuales en los que el contenido se enfoca a todo tipo de público interesado en cuestiones diversas y relacionadas con la historia o las humanidades. A esto hay que unir el hecho de que las redes sociales han favorecido diferentes formas de participación individual y colectiva, cambiando por completo la forma en la que la historia se muestra y llega a la sociedad (NOIRET, 2018: 119). En cualquier caso, esta situación en la que la divulgación histórica se ha insertado en las redes sociales y la web en general a través de diferentes formatos tiene varios problemas. Como indica Serge NoIRET (2018: 115): «Es necesario, casi habría que decir esencial, hacer un análisis a fondo del contenido siempre que buscamos información en la red y tratamos con textos de redes sociales». Y es que tanto las redes sociales como los diferentes formatos de divulgación web son plataformas en las que se construye una divulgación colectiva que no siempre se basa en una metodología rigurosa o información adecuada. Esto no es realmente un problema de la historia digital, más bien tiene que ver con el uso que se les da a las redes y con la usual confusión entre divulgación histórica de calidad y algo que podría definirse como "fake history», que ha pasado de ocupar espacios en librerías y bibliotecas a estar más accesible en diferentes plataformas virtuales y redes sociales. 
Pese a la situación descrita, más frecuente de lo que parece, también existen diversos proyectos de blogs, cuentas de redes sociales y otro tipo de webs que realizan una divulgación que respeta el rigor histórico y fundamentan sus afirmaciones en evidencias empíricas y fuentes históricas. A su vez, cada vez es más habitual el uso de plataformas de vídeo o streaming como YouTube o Twitch, indicadores muy importantes que reflejan cómo la historia digital está adaptando sus herramientas y metodologías de investigación y divulgación a la nueva realidad que presentan las sociedades humanas superando las carencias que presentaba hace unos años la distinción entre el «modelo tradicional académico» y «modelo social» (SPENCE, 2014: 124-126).

En esencia, hoy la historia digital supone una forma constantemente innovadora de trabajar con la historia, que tiene una gran capacidad para generar proyectos o trabajos en los que se producen nuevas narrativas históricas o se completan las existentes, ofreciendo posibilidades para ampliar las memorias colectivas y generando espacios en los que tienen cabida los relatos subalternos. Las cuestiones mencionadas, junto a su constante capacidad de adaptación e innovación, convierten a esta forma de hacer historia en una de las más relevantes en la actualidad y la hacen contar con un prometedor futuro en los próximos años.

\section{EL MEMORICIDIO EN EL CASO PALESTINO}

En 1948 tuvo lugar la llamada Nakba o «catástrofe» palestina, resultado de una serie de dinámicas coloniales ejecutadas durante el Mandato Británico e impulsadas por el movimiento sionista que pretendía establecer un estado judío en lo que era la Palestina histórica (BASALlote, 2015, 33). Las fuentes señalan que entre 418 y 614 localidades palestinas fueron borradas del mapa o repobladas desde finales de 1947 hasta finales del siguiente año, y aproximadamente entre 750.000 y 800.0000 personas fueron expulsadas por las políticas sionistas de terror y consiguieron huir y refugiarse en los países árabes fronterizos (BASALLOTE et al, 2017: 38). El historiador israelí Ilan Pappé determinó que esas políticas respondían a un plan que pretendía la limpieza étnica de Palestina, expulsando a su población autóctona para crear un estado judío (PAPPÉ, 2006).

La Nakba desafió la propia existencia de la población palestina, de su identidad y de su memoria histórica puesto que desató una serie de dinámicas que desplazaron forzosamente a la población autóctona, facilitaron el avance de la colonización del territorio y la sustitución de la población palestina por población alóctona. Y, sin embargo, la historiografía israelí ha negado sistemáticamente la existencia de este fenómeno que cuestionaba el modo en el que se había producido la fundación del Estado de Israel hasta la aparición de posiciones críticas por parte de los nuevos historiadores israelíes en la década de 1980 (GIJÓN MENDiguTía, 2008).

La invisibilización de la Nakba fue acompañada de la negación del derecho al retorno de las personas que se habían convertido en refugiadas (MASALHA, 2003) y la negación de su identidad palestina por un Estado que no reconoce su existencia diferenciada de la identidad árabe (BARREÑADA BAJO, 2005). El constante interés del movimiento sionista por borrar del mapa cualquier indicio de la existencia de un pueblo y una tierra llamada Palestina llevó incluso a la formación de comités 
para renombrar sistemáticamente cada topónimo y cada punto de referencia en el paisaje del estado recién creado y desarrollar nuevos mapas hebreos (BENVENISTE, 2000: 11-54). Historiadores como Ilan PAppé (2006) o Nur MAsalHa (2003 y 2012) han denominado a este proceso como memoricidio.

El memoricidio es un término que conceptualiza actos específicos de destrucción de lugares de la memoria y artefactos culturales, religiosos y étnicos para erradicar el recuerdo de eventos pasados e identidades (HARAČíć, 2012). Fue acuñado por el médico e historiador croata Mirko D. Grmek en 1992, para «definir la destrucción intencional de la memoria y el tesoro cultural del «otro», del adversario, del (des)conocido», durante las guerras de la antigua Yugoslavia (BlAžINA, 1996). Entendido así, el memoricidio se puede entender como exterminación cultural o, incluso, como genocidio cultural (HARAČIĆ, 2012: 237). A lo largo de la historia, se ha manifestado principalmente a través de tres fenómenos: la destrucción del patrimonio histórico y cultural, la destrucción de libros y la denegación de la propia historia junto a la imposición de una narrativa histórica externa.

En el caso de la población palestina, la destrucción del patrimonio histórico y cultural ha sido una constante desde la Nakba ya que el Estado de Israel, que nace en ese momento, intentó desarabizar el territorio palestino por todos los medios posibles perpetrando un memoricidio cultural. Así, las localidades palestinas desalojadas en 1948-1949 fueron metódicamente destruidas a lo largo de los años siguientes y hasta la década de 1960, ya fuera totalmente $(70 \%)$ o parcialmente $(22 \%)$. Solo siete sobrevivieron, siendo ocupados mayoritariamente por personas judías israelíes. El resto de áreas que fueron destruidas, poco después se convirtieron en zonas de cultivo, parques arqueológicos o aparcamientos y borraron los restos de la presencia física de la población autóctona sobre ese territorio (BASAllote MaRín et al., 2017: 63).

Además, el patrimonio histórico religioso se vio afectado por esta destrucción puesto que el proyecto colonial israelí también reconsagró santuarios musulmanes, que nunca habían sido parte de la tradición judía, como santuarios judíos. Esta política de apropiación cultural y religiosa llevó a renombrar las tumbas de personas consideradas santas y lugares sagrados musulmanes como lugares judíos a los que se asignaron nombres de resonancia bíblica (MASALHA, 2012: 112-113).

Además, la estrategia de judaizar el territorio transformó los ecosistemas de la región para eliminar el vínculo entre la tierra y la población autóctona palestina. Así, por ejemplo, «el Fondo Nacional Judío (FNJ en adelante) sustituyó especies de la flora autóctona (olivos, almendros, higueras, chumberas, algarrobos...) por árboles europeos (especialmente el pino) en estos nuevos bosques» (BASALLOTE et al., 2017: 64). Esta estrategia ha continuado en las siguientes décadas de manera que, en otro ejemplo de finales de la década de 1960, el FNJ decidió plantar alrededor de un millón de árboles para «rodear Jerusalén con un cinturón verde» prometiendo de este modo lugares únicos a las personas visitantes (PAPPÉ, 2006), pero sin tener en cuenta los costes medioambientales que tendría la sustitución de la flora autóctona por esta medida y su impacto en el ecosistema y en los medios de subsistencia tradicionales de la población palestina.

Otra de las manifestaciones históricas del memoricidio, como es la destrucción de libros, también ha estado presente en la realidad palestina. Así, bibliotecas y archivos han sido objeto de destrucción por agentes israelíes que han eliminado o 
se han apropiado los libros, documentos y registros conservados en esos lugares. Por ejemplo, las bibliotecas de las personas palestinas que fueron expulsadas durante la Nakba fueron expoliadas y el Estado israelí se apropió de muchos de sus fondos. Parte de dichos fondos fueron donados a las escuelas árabes, otros fueron entregados al Departamento de Estudios Orientales de la Librería Nacional, pero, alrededor de 26.000 libros fueron declarados inadecuados para su uso en las escuelas árabes de Israel o para su venta, porque podían alentar comportamientos que amenazasen al Estado, y fueron vendidos como papel desechable en 1957 (АмIт, 2008).

Cuando Israel invadió el Líbano, en el año 1982, se apropió de los archivos del Palestine Research Center y del Palestinian Cinema Institution, instituciones que se encontraban en Beirut en aquellos momentos. Mientras que el primero fue devuelto tras ser copiado, el otro, que contenía material audiovisual, se ha mantenido en el archivo de las Fuerzas de Seguridad Israelíes hasta la actualidad (SELA, 2017).

A ello se puede añadir la destrucción generada en librerías, archivos e instituciones culturales por el ejército israelí durante sus incursiones militares en las comunidades palestinas. Por ejemplo, durante el periodo de la segunda intifada, fueron significativos los daños realizados en 2002 al Centro Cultural Khalil Sakatini, en Ramallah, que era la sede de una biblioteca y de la editorial de la prestigiosa revista Al-Karmel. Se perdieron registros de la propiedad de la tierra, los historiales de más de un millón de estudiantes de enseñanza primaria, secundaria y superior, registros policiales, etc. En aquel periodo también resultaron dañadas bibliotecas de la Universidad de Al-Quds en Al-Bireh y de la Universidad de Bethlehem (BÁEZ, 2004: 262).

La denegación de la propia historia junto a la imposición de un discurso histórico externo es la última de esas manifestaciones históricas del memoricio. En el caso que nos ocupa podemos encontrar múltiples ejemplos de esa imposición de una narrativa histórica hegemónica, que constituía una verdad oficial y que no fue cuestionada por la historiografía israelí hasta fechas recientes, reproduciendo los mitos construidos por el poder colonial. De esta manera se ha silenciado el pasado palestino, negándole su propia historia, hasta llegar a situaciones tan dramáticas como la aprobación por la Kneset, en el año 2001, de la ley que prohibía la conmemoración de la Nakba en el Estado de Israel, donde más de un millón de personas palestinas son ciudadanas del mismo:

En la práctica esto significa que si un teatro, una escuela o una universidad, por ejemplo, permiten que ciudadanos palestinos realicen una vigilia o una protesta para hablar sobre la catástrofe de 1948, en contraste con el día de la Independencia de Israel, el gobierno puede amenazar a esas instituciones con retirarle los fondos (BERMÚdeZ, 2021).

$Y$ es que Israel no solamente ha robado o saqueado los elementos del patrimonio palestino físicamente. También:

Ha creado un estricto sistema de gestión, control y producción del conocimiento que incluye leyes, reglas, normas, métodos, procedimientos de archivo tales como la censura, el estudio restringido, la prohibición/limitación del acceso, el control 
sobre lo que se clasifica (para quién y hasta qué grado), catalogación y etiquetado de acuerdo a los códigos y a la terminología sionista que difiere de la terminología original palestina, significando una propiedad israelí sobre el material (SELA, 2017: 202).

La persistencia de estos mecanismos de control se ha mostrado también en el acceso que el Estado de Israel proporciona a sus propios fondos documentales relacionados con la Nakba. Así, a mediados de la década de 1980 comenzó la desclasificación de parte de la documentación siguiendo la Ley de Archivos de 1955, lo que permitió el acceso a registros de los Archivos Ben Gurión, del Estado de Israel, de las Centrales Sionistas, de la Haganá, de las Fuerzas de Defensa Israelíes o de los Yad Yaari del que se beneficiaron los llamados nuevos historiadores israelíes. Sin embargo, otra parte muy importante de la documentación sobre 1948 nunca se desclasificó y se mantuvo inaccesible (RAmos TolosA, 2020: 55). Más tarde, desde mediados de la segunda década del siglo XXI, muchos de los fondos israelíes relacionados con la Nakba que ya habían sido desclasificados, dejaron de estar disponibles para consulta, lo que ha sido interpretado por el prestigioso historiador israelí Ilan Pappe, como un intento de cubrir los crímenes contra la población palestina en 1948 (PAPPÉ 2020).

De esta manera se ha pretendido reforzar las narrativas sionistas. Sin embargo, la población palestina ha carecido de un sistema similar para construir un conocimiento propio sobre el que desarrollar sus discursos históricos, ante la presión colonial y la ausencia de un Estado propio. En la actualidad, el desarrollo de proyectos de historia y humanidades digitales está corrigiendo esta situación, como veremos en el siguiente apartado, creando espacios virtuales accesibles para los investigadores palestinos que, en muchos casos, no tenían acceso anteriormente a su propia documentación histórica, como explica la investigadora Lauren BANKO (2012).

El profesor Jorge RAmos Tolosa definía el memoricidio como «la eliminación física de los elementos identitarios del pueblo palestino, el intento de borrar su paso por la tierra y la pretensión de acabar con su memoria colectiva» (2015: 164). Por lo que sostiene la idea de que, a pesar de la traumática experiencia que ha supuesto la Nakba palestina y el memoricidio que la ha acompañado, el fenómeno de la Nakba ha sido clave para el desarrollo de la identidad palestina y de las resistencias de esta población a las dinámicas colonizadoras. Ello se ha comprobado, por ejemplo, con su reiterada presencia en las diferentes estrategias del pueblo palestino para reivindicar el derecho al retorno, reconocido internacionalmente por la Resolución 194 de la Asamblea General de las Naciones Unidas.

\section{EL USO DE LA HISTORIA DIGITAL PARA LA RECUPERACIÓN DE LA MEMORIA HISTÓRICA PALESTINA}

La historia digital está permitiendo que la memoria histórica y colectiva del pueblo palestino sea recuperada. Más allá de este proceso de recuperación, se ha puesto en valor, difundido, enseñado y transferido gracias a los medios y 
metodologías que las nuevas tecnologías posibilitan.

Del conjunto de iniciativas identificadas que intentan recuperar la memoria histórica palestina, mediante proyectos relacionados con la historia digital, se han seleccionado tres para este estudio. Cada uno de ellos cuenta con diferentes recursos, estrategias y metodologías para contribuir a la resistencia de la población palestina frente al memoricidio.

Todos ellos destacan, principalmente, por centrar su atención en los momentos previos y posteriores a la Nakba, evitando así que este punto de inflexión para la población palestina y para la historia de Palestina-Israel caiga en el olvido y desaparezca fruto de la influencia del discurso hegemónico que se propone desde la historiografía sionista.

\subsection{Palestinian Oral History Archive}

El primero de los casos que vamos a analizar es el Palestinian Oral History Archive $^{2}$ (POHA en adelante), que ofrece una buena muestra de las posibilidades de la historia digital para la preservación de la memoria colectiva y para proporcionar fuentes primarias relevantes para un análisis riguroso de la historia. En concreto, el POHA ha contribuido a ampliar y renovar la historiografía de la Nakba.

Los orígenes del proyecto se iniciaron ante la evidencia del envejecimiento de la población debido al tiempo que había pasado desde 1948-49 y la necesidad de preservar el testimonio de la generación que había asistido a su expulsión de la Palestina histórica. Nació fruto del esfuerzo del trabajo del Centro de Recursos Árabes para las Artes Populares (1994-2009) y del Archivo Nakba (2002-2006) que entrevistaron de manera sistemática a miembros de la primera generación de la comunidad palestina refugiada en Líbano durante la Nakba, creando una base de datos con más de 800 testimonios en video y/o audio que, posteriormente, fue consolidada en la colección del POHA (Sleiman y CHEBARO, 2018).

A partir de 2011, estas organizaciones contactaron con el Instituto Issam Fares de Políticas Públicas y Asuntos Internacionales de la Universidad Americana de Beirut para poner en marcha el POHA, lo que se traduce al castellano como el Archivo de Historia Oral Palestino.

Se trata de una colección de archivos que contiene más de 1.000 horas de testimonios con personas refugiadas, principalmente de primera generación y que tiene como objetivo ampliar las colecciones de historia oral que documentan diversos aspectos de la experiencia palestina en el Líbano y en otras partes la región. Para ello, los testimonios orales grabados en audio y/o video fueron digitalizados, indexados, catalogados y preservados, facilitándose el acceso público a este material mediante la creación de una plataforma digital.

Los testimonios de las personas palestinas que vivieron de primera mano la Nakba, y que se convirtieron en personas refugiadas, son fuentes primarias fundamentales para la reconstrucción de la historia, geografía y cultura palestina, anterior a 1948. El archivo documenta las historias de vida de personas palestinas que residen en campos de refugiados y en diferentes comunidades del Líbano. En la web del mismo se indica que «El enfoque principal del Archivo son los relatos

2 La página web del Palestinian Oral History Archive puede consultarse en: https://libraries.aub. edu.lb/poha/ 
personales que rodean la Nakba, que aclaran un momento decisivo en la historia y la experiencia colectiva palestinas», lo que refuerza la identidad palestina.

El POHA ha organizado sus fondos en cuatro colecciones: 1. "Desarraigo», que recoge la mayor parte de los testimonios y se centra en la experiencia de expulsión masiva de la población refugiada durante la Nakba; 2. "Cuentos populares», que comprende elementos de la cultura anterior a 1948 en forma de cuentos y canciones populares palestinas, poemas, etc.; 3. «Ayn al-Hilwat» que recoge vídeos de mujeres del campo de personas refugiadas de Ayn al-Hilwah, situado en el sur del Líbano, donde hablan de varios temas como sus ocupaciones, vidas familiares y su papel en el establecimiento del campo; y 4. «Biografías», que registran las historias de vida de hombres y mujeres que desempeñaron papeles importantes en sus comunidades.

La base de datos que da acceso a las fuentes orales es sencilla de utilizar y está disponible tanto en árabe como en inglés, lo que facilita el acceso a la información por un mayor número de usuarios. La búsqueda de los testimonios se puede realizar a través de las diferentes colecciones existentes y seleccionando alguna de las categorías creadas para organizar los registros (título, tema, fecha, entrevistado/a, lugar de origen y punto de referencia).

Cada entrevista proporciona una información muy completa, presentando una breve biografía de la persona entrevistada, su nombre, la duración de la entrevista, la colección a la que pertenece, el idioma en el que se realiza y las temáticas que se abordan en ella, entre otras cuestiones. En algunos casos, la propia entrevista aparece traducida al inglés.

POHA nos ofrece además una sección llamada «mapa interactivo», donde podemos ver cartografías históricas de Palestina en la década de 1940 y una imagen por satélite de la Palestina histórica en 2019. En ambos casos se pueden apreciar las ciudades, aldeas y puntos de interés como instituciones culturales y turísticas, monumentos, etc. Estos recursos son el resultado de la colaboración de este proyecto con el de Palestine Open Maps. ${ }^{3}$

Este mapa interactivo permite asociar a las personas entrevistadas a su localidad de origen que hoy ya no existe, representando no solamente un punto físico geográfico, sino también temporal, anterior a la Nakba, reforzando su identidad compartida de personas refugiadas, visibilizando el vínculo con la tierra de origen y facilitando el mantenimiento del sentimiento de pertenencia a una comunidad palestina que atravesó por un fenómeno histórico que le ha marcado hasta la actualidad y que comparte el deseo de retornar a Palestina.

Podemos destacar la importancia de las colecciones que ofrece el proyecto POHA porque recogen unas fuentes primarias orales que completan unas fuentes oficiales y registros escritos extremadamente escasos y dispersos en el caso palestino. Así, «los testimonios orales de la Nakba ayudan a construir una narrativa de pérdida «de adentro», una muy útil y potente herramienta en busca de justicia para las comunidades palestinas» (SLEIMAN y CHEBARO, 2018: 63). Además, metodológicamente, da acceso, voz y legitimidad a narrativas en primera persona y a perspectivas de poblaciones marginalizadas (personas sin tierra, campesinas, mujeres, refugiadas o pobres), constituyendo un archivo digital que recoge las necesidades y experiencias de las comunidades a nivel de base (Sleiman y Chebaro, 2018).

3 PaLestine Open MAPs (2019): https:/ / palopenmaps.org [consulta: 15/07/2021]. 
Este proyecto es el resultado de un interesante trabajo interdisciplinar en el que ha participado prestigiosas personas del ámbito académico, bibliotecario y profesional, de diferentes disciplinas y orígenes, lo que ha enriquecido los productos que ha elaborado. Las entrevistas fueron realizadas por varias instituciones empleando diferentes técnicas para su elaboración, lo cual no fue un impedimento a la hora de la construcción del POHA, sino que enriqueció su contenido y permitió aprovechar las sinergias de las distintas iniciativas a partir de su colaboración.

El proyecto permite un acceso abierto a su base de datos para que todas las personas interesadas, de cualquier sector, puedan consultar los testimonios. Por ello, cuenta con una plataforma digital de uso gratuito que puede ser utilizada tanto por investigadores académicos, activistas, profesionales y por cualquier miembro de la propia comunidad palestina interesada en conocer esa memoria que se ha intentado borrar a través del memoricidio.

Por tanto, el trabajo que hacen todas las entidades colaboradoras en el proyecto es fundamental para preservar la memoria colectiva del pueblo palestino en torno al fenómeno de la Nakba. Quizá, más adelante, este archivo pueda extender su alcance y recoger las experiencias de las personas palestinas refugiadas en el Líbano en 1967, lo cual sería muy interesante para la lucha contra el memoricidio de la Naksa, ${ }^{4}$ aunque requeriría la inversión de mayores recursos en el proyecto.

\subsection{Zochrot Nakba Map}

El segundo caso que presentamos es el de Zochrot, una ONG que ha creado un espacio virtual para construir y divulgar el conocimiento en torno a la Nakba palestina en la sociedad israelí. Zochrot toma su nombre de una palabra hebrea que significa «recordar». La organización tiene su sede en Tel Aviv y fue fundada para facilitar la comprensión de la Nakba palestina a la población israelí no árabe y que se expresaba en hebreo, dado que, como hemos mencionado anteriormente, había sido un proceso ampliamente silenciado en la historiografía y del que la sociedad israelí contemporánea apenas era consciente.

La mayoría de sus impulsores estaban ya implicados en proyectos educativos dirigidos a mejorar la coexistencia y la comprensión mutua entre palestinos y judíos en Israel (MorocutTI, 2013: 152). En ese contexto, cuando aún parecía estar vivo el Proceso de Oslo y las negociaciones de paz entre el Estado de Israel y los representantes palestinos, Zochrot se propuso transformar la visión mayoritaria de la sociedad israelí que, influida por la historiografía tradicional, seguía descuidando y pasando por alto la implicación de las milicias e instituciones sionistas en la expulsión y limpieza étnica de la mitad de la población palestina que habitaba la Palestina histórica en 1948, negaba las dimensiones de la Nakba y defendía las políticas estatales que impedían el retorno de las personas refugiadas y seguían generando sufrimiento. Una tarea nada sencilla, pero que entendía necesaria para cambiar la posición mayoritaria de la opinión pública israelí sobre los acontecimientos de 1948-1949 y, así, facilitar la reconciliación entre las dos comunidades.

4 El término Naksa se traduce al castellano como «recaída». Así es como se le denominó a la Guerra de los Seis Días o Guerra de 1967, en la que Israel ocupó militarmente Gaza, Cisjordania, Jerusalén Este, los Altos del Golán y el Sinaí. 
Las actividades de Zochrot se despliegan en el mundo físico y en el mundo digital. La organización ha generado una base de datos con los registros de pueblos palestinos destruidos desde 1948 y los nombres de las localidades israelíes que fueron construidos en sus tierras, a partir de testimonios orales recogidos por la organización y de la documentación cartográfica existente. Esta información ha sido georreferenciada en un mapa digital que incluye los pueblos destruidos y diferentes detalles sobre cada uno de ellos, para disputar la representación geográfica hegemónica israelí. Así, los registros contienen el nombre del pueblo o ciudad, sus coordenadas geográficas, datos sobre la población judía previa a la Nakba y sobre las colonias judías establecidas en esos lugares posteriormente. Incluye una breve descripción de la historia de la localidad, antes y después de 1948, con referencias bibliográficas a distintas fuentes. Además, los registros se completan con imágenes de las poblaciones, vídeos que contienen entrevistas y testimonios de residentes o refugiados que huyeron de esos lugares e incluso folletos en formato digital producidos a partir de visitas sobre el terreno durante el que se recogió información gráfica o audiovisual. ${ }^{5}$ Estas visitas a las localidades palestinas destruidas, que comenzaron en 2003, están abiertas a quienes quieran participar y se documentan audiovisualmente, quedando los documentos a disposición de los usuarios que quieran consultarlos en la web de la organización. ${ }^{6}$

Estas visitas y los procesos de documentación e intercambio que generan permiten, según Zochrot, establecer una memoria histórica colectiva a partir de las experiencias de las personas palestinas y judías participantes. Así, para las personas palestinas este evento es un viaje atrás en el tiempo al lugar donde solían vivir. Para las personas judías, la visita y la conmemoración de esos lugares revelan unos recuerdos que están ocultos a la vista. Los recuerdos revelados a menudo compiten con el recuerdo común, sionista del lugar. Pero es que, además, estas visitas constituyen acciones públicas simbólicas que permiten reafirmar la existencia de las poblaciones palestinas destruidas dentro del paisaje y la memoria israelí, dejando de ser parte de un pasado silenciado.

En el año 2014, Zochrot lanzó una aplicación para teléfonos móviles llamada iNakba para aumentar la sensibilización de la sociedad israelí sobre la Nakba (KLEIN, 2014). La aplicación facilita la accesibilidad a la base de datos y, además, permite a sus usuarios colaborar con la organización para mejorar y ampliar la información disponible en la base de datos compartiendo sus fotografías, vídeos, actualizaciones o correcciones. ${ }^{7} \mathrm{La}$ aplicación, basada en tecnología de navegación GPS, utiliza Google Maps and Waze como plataformas para permitir a los usuarios encontrar esas localizaciones, aprovechando el enfoque metodológico del mapeo digital y las capas que superpone la aplicación para sortear el hueco que existe entre el pasado y el presente (MusiH y FISHER, 2021). Es una aplicación trilingüe (hebreo, árabe e inglés) y está disponible para sistemas Android y Apple.

El impacto de la actividad de Zochrot en el mundo digital es significativo y puede medirse a partir de la extensión del uso de sus herramientas digitales, tanto mediante el estudio del número de usuarios de la página web, que permite

\footnotetext{
5 En este enlace se puede consultar la información registrada para la entrada de «Haifa»: https:// zochrot.org/en/village/49192

6 Se puede acceder a los videos de las visitas a los pueblos destruidos durante la Nakba en: https:// zochrot.org/en/tour/all

7 La aplicación iNakba puede encontrarse en el siguiente enlace: https://zochrot.org/en/keyword/45323
} 
acceder, entre otras cosas, a la documentación contenida en la base de datos, como mediante el cálculo del número de descargas de la aplicación iNakba en teléfonos móviles y tablets. Así, el número de usuarios únicos que visitaron su página web durante 2019 fue de 96.411, procediendo de Israel la mitad de ellos. Esa es una tendencia que se mantiene si se extiende el análisis durante el periodo 20172019, cuando 212.622 israelíes accedieron a la página web. En cuanto al uso de la aplicación iNakba, desde su lanzamiento y hasta 2019, el número de descargas era superior a 50.000 y los usuarios activos alcanzaba los 5.344, participando casi 600 de ellos en acciones colaborativas a través de la aplicación (ZоснROT, 2020).

De esta manera y tras dos décadas de trabajo, Zochrot ha conseguido que su base de datos se haya convertido en el archivo más completo y accesible para el conocimiento de la Nakba en hebreo. Un archivo que se sigue completando y ampliando de manera colaborativa año tras año por una comunidad de usuarios y que, como la mayoría de materiales y productos creados por Zochrot, son fácilmente accesibles a partir de descargas gratuitas desde su página web.

\subsection{Palestine Open Maps}

El tercer caso que presentamos es el del proyecto Palestine Open Maps, un claro ejemplo de cómo la historia y las humanidades digitales pueden contribuir a la preservación de la memoria colectiva y a la elaboración de narrativas históricas alternativas fundamentadas que cuestionan los discursos hegemónicos o coloniales, como en el caso de Palestina-Israel. Palestine Open Maps es un proyecto de cartografía digital que nació en 2018 fruto de la colaboración entre Visualizing Palestine y la Universidad de Columbia (Studio-X Amman). Tiene como principal objetivo ofrecer en acceso abierto un conjunto detallado de mapas históricos del periodo del Mandato británico de Palestina, combinándolos con otras fuentes de datos disponibles para facilitar la comprensión de las transformaciones ocurridas en la geografía humana de la Palestina histórica en el último siglo.

La idea de Palestine Open Maps se inspiró en el proceso de digitalización de una colección de mapas topográficos de época del Mandato británico llevado a cabo por la Biblioteca Nacional de Israel. La relevancia de este trabajo de digitalización radicaba en que esta colección de mapas pasó al dominio público, dejando de ser documentos históricos difícilmente accesibles y ofreciendo una relevante perspectiva de los cambios geográficos, políticos, naturales o humanos del territorio de Palestina-Israel. Esta cuestión hizo que se pusiese en marcha el proyecto Palestine Open Maps, para digitalizar y hacer más accesibles 155 mapas históricos de Palestina-Israel a escala 1:20.000, que cubren la parte más poblada de la región, desde el sur en Beersheba hasta las fronteras del norte con Líbano y Siria. Además, se digitalizaron otros mapas menos detallados a escala 1:100.000 y 1:250.000 que cubrían todo el país, y otros de mayor escala que mostraban al menos veinte pueblos y ciudades importantes con gran nivel de detalle (BARCLAY, 2020).

La digitalización de estos mapas históricos proporciona una fuente útil para las investigaciones de los profesionales de la historia puesto que proporciona un testimonio que las reproducciones digitales disponibles, como el Atlas of Palestine de Salman Abu Sitta (2010) o la aplicación de la iNakba de Zochrot, no pueden ofrecer. Sin embargo, su utilidad seguiría siendo limitada para el 
resto de la sociedad. Por ello, el proyecto Palestine Open Maps va más allá e incorpora otros recursos de acceso libre que existen online, incluyendo fotografías históricas y testimonios orales, ampliando el uso que se puede hacer de los mapas y facilitando la visualización de la información a través de una web interactiva.

El resultado principal del proyecto ha sido una plataforma en constante desarrollo que combina diferentes tecnologías aplicadas al mapeo y la narración inmersiva. Es relevante señalar que todo el contenido de Palestine Open Maps es abierto y gratuito. A su vez, destaca cómo el proyecto ha ido adquiriendo mucho más contenido e información con el paso del tiempo, gracias a su carácter colaborativo, con las aportaciones y al trabajo realizado por investigadores, periodistas y representantes de la sociedad civil. Destacan las contribuciones de datos registrados y digitalizados previamente por dos entidades ya mencionadas (Palestine Land Society y Zochrot) y de otras como Palestine Remembered.

Cabe señalar que la principal limitación de Palestine Open Maps es que solo se circunscribe al periodo que va desde 1870 hasta 1951, pudiendo realizarse una comparativa con mapas de 2019. Esto quiere decir que el espacio cronológico que queda por incluir es bastante amplio y relevante, sobre todo si tenemos en cuenta los diferentes cambios y acontecimientos que han tenido lugar en el contexto de Palestina-Israel entre 1952 y 2021. Esta situación puede cambiar a medio plazo, pues uno de los objetivos del proyecto es el de ampliar sus funciones web, su utilidad y su contenido.

En esencia, Palestine Open Maps nos permite conocer una serie de cambios y transformaciones tales como los primeros momentos de colonización sionista, los últimos momentos de gobierno otomano y las transformaciones producidas por la constitución del Mandato británico o las consecuencias de la Nakba. Una serie de procesos que se pueden observar a través de mapas históricos digitalizados e interactivos, en los que además de realizar comparaciones y observar diferentes cambios se puede conocer un poco más de diferentes localizaciones de relevancia para el estudio de la cuestión de Palestina.

Lo más destacable de este proyecto es su contribución a la preservación de la memoria colectiva y la identidad del pueblo palestino frente a los procesos colonizadores y las prácticas memoricidas del Estado de Israel. En primer lugar, al facilitar el acceso a esos documentos históricos, permite que sean fácilmente desmontados algunos mitos de la narrativa sionista que, por ejemplo, afirmaba la inexistencia de la población palestina sobre el territorio repitiendo el mantra: «Un pueblo sin tierra para una tierra sin pueblo». Palestine Open Maps, en segundo lugar, también permite observar el antes y el después de la Nakba palestina, mostrando los cambios poblacionales que ocurren en las diferentes localizaciones como consecuencia de la colonización y la limpieza étnica de 1948. Se visibiliza el alcance de la catástrofe mediante la identificación de los pueblos despoblados y la construcción de nuevas colonias judías en el territorio, en muchos casos sobre las propias ruinas de poblaciones palestinas. En tercer lugar, y no menos relevante, es su registro de decenas de miles de nombres de topónimos, todos ellos sistemáticamente transliterados del árabe, que incluyen las poblaciones existentes, montañas, valles y ríos, así como otros puntos de interés como huertas, depósitos de agua o santuarios. Su importancia radica en constituir una evidencia de la realidad existente anterior a 1948 y disputar las narrativas hegemónicas israelíes que utilizaron las herramientas cartográficas a partir de 1941 para crear mapas hebreos del nuevo país que renombraron cada topónimo e invisibilizaron 
a la población y cultura autóctona (BARCLAY, 2020: 178-179).

No puede obviarse que los mapas o documentos cartográficos reflejan cambios históricos que constituyen un elemento de relevancia en la memoria colectiva, mucho más evidentes en el contexto de Palestina-Israel donde las disputas y la legitimación territorial han sido ejes de conflicto a lo largo del tiempo. El hecho de preservar, difundir y hacer accesibles documentos como los disponibles en Palestine Open Maps permite proporcionar evidencias para un estudio riguroso de la evolución histórica de Palestina-Israel. Además, este proyecto muestra cómo las humanidades digitales están cuestionando los modelos tradicionales de construcción del conocimiento hegemónico, pues facilitan a grupos subalternos el acceso a herramientas y metodologías que permiten desafiar las narrativas oficiales y crear otras cartografías que mapeen narrativas alternativas a las tradicionales.

\section{CONCLUSIONES}

La memoria histórica y colectiva de la población palestina ha sufrido las graves consecuencias de unas políticas coloniales que han ejercido una notable violencia contra ella y han supuesto el desarrollo continuado de un memoricidio desde 1948. Ante esta situación, la historia digital supone una forma de contrarrestar este proceso de eliminación y transformación histórica y cultural, cuestionando las narrativas hegemónicas respecto a la Nakba y la cuestión de Palestina.

Los proyectos analizados muestran las posibilidades de la historia digital para recuperar y preservar la memoria histórica palestina, así como para construir discursos alternativos que resultan de las necesidades y realidades de las poblaciones subalternas, sin necesidad de estar mediados por actores institucionales que respondan a lógicas de arriba hacia abajo. A pesar de sus diferencias, los distintos proyectos comparten una línea de trabajo común como es la de recuperar la historia palestina y la centralidad de la Nakba en la misma. De manera que, aprovechando las posibilidades que les ofrecen las humanidades digitales, desarrollan procesos que van más allá de la digitalización de las fuentes documentales, generando procesos de colaboración que les acercan a las propuestas de la ciencia ciudadana, buscando construir un conocimiento compartido a partir de las experiencias individuales y colectivas de los usuarios.

Los proyectos han puesto en valor fuentes primarias y secundarias para el estudio de la historia palestina, que van más allá de la simple acumulación de documentos escritos, gráficos, orales, cartográficos o audiovisuales, y permiten vincular múltiples registros a través del uso de bases de datos e hipertextos para conectar la información. Además, para una población que ha sido expulsada en gran parte de su territorio, las herramientas de cartografía y mapeo digital, cobran una relevancia importante puesto que permiten la construcción de capas de información con una manifiesta carga política.

También resulta necesario señalar cómo estas propuestas, que derivan de proyectos que se sitúan en el ámbito de las humanidades digitales, se aprovechan de las prácticas colaborativas y de acceso abierto de sus procesos de generación del conocimiento para desarrollar propuestas interdisciplinares que incorporan distintas áreas de saberes, pero que también conectan a sectores muy diversos de la población palestina, que cooperan para elaborar esas narrativas históricas y, de esa manera, las enriquecen y completan, atendiendo a dinámicas, enfoques o 
actores a los que la historiografía más tradicional no presta tanta atención. Además, estos proyectos destacan por su capacidad de continua innovación y adaptación a las nuevas realidades, lo que les permite navegar entre las limitaciones y las posibilidades que les ofrecen los cambiantes entornos políticos y tecnológicos. Una buena muestra de ello es el desarrollo de la aplicación iNakba por Zochrot.

En conjunto, los proyectos aquí analizados evidencian la notoria apuesta por la divulgación que la historia y las humanidades digitales realizan. De manera que el fruto de sus trabajos e investigaciones se traduce en una activa labor pedagógica y de difusión del conocimiento generado a la sociedad tanto en Israel, como en Líbano y al resto de la comunidad internacional, para preservar la memoria de la población palestina, fortalecer sus narrativas históricas y luchar contra las políticas memoricidas.

\section{REFERENCIAS}

Амiт, G. (2008): «Ownerless objects? The story of the books Palestinians left behind in 1948», Jerusalem Quarterly: Plundering Palestine, 33: 7-20.

BÁEz, F. (2004): Historia universal de la destrucción de libros. De las tablillas sumerias a la guerra de Irak, Ediciones Destino, Barcelona.

BANKO, L (2012): «Occupational hazards, revisited: Palestinian historiography», Middle East Journal, 66 (3): 440-452.

BARClay, A. (2020): «Mapping Palestine: Erasure and unerasure», en G. CARABelli, M. Jovanović, A. Kirbis y J.F. WALton (eds.), Sharpening the haaze. Visual essays on imperial history and memory, Ubiquity Press, London: 177-189.

BARREÑADA BAJO, I. (2005): Identidad y ciudadanía en el conflicto israelo-palestino: los palestinos con ciudadanía israeli, parte del conflicto y excluidos del proceso de paz, Tesis Doctoral, Universidad Complutense, Madrid.

Basallote Marín, A. (2015): La Cuestión Israelí: Sionismo y Disidencia. Ideología, Identidad y Contestación Social en la Sociedad Judía de Israel, Tesis Doctoral, Universidad de Sevilla, Sevilla.

Basallote Marín, A.; Checa Hidalgo, D.; lópez Arias, L.; Ramos Tolosa, J. (2017): Existir es resistir: pasado y presente de Palestina-Israel, Editorial Comares, Granada.

Benvenisti, M (2000): Sacred Landscape: The Buried History of the Holy Land Since 1948, University of California Press, Berkeley.

BERMúdeZ, A. (2021): «Conflicto israelí-palestino: las controvertidas leyes que los ciudadanos árabes en Israel denuncian como discriminatorias», $B B C$. https://www.bbc.com/mundo/noticiasinternacional-57209473 [Consulta: 05/07/2021].

BLAŽINA, V. (1996): «Mémoricide ou la purification culturelle: la guerre contre les bibliothèques de Croatie et de Bosnie-Herzégovine», Documentation et bibliothèques, 42: 149-164.

BoCANEGRA BARBECHO, L. (2021): «Fuentes digitales para el estudio del exilio republicano español», Hispania Nova, vol. 1 extraordinario: 12-46. https:// doi.org/10.2307/25095630

Cohen, D.J.; Rosenzweig, R. (2006): Digital History: A Guide to Gathering, Preserving and Presentig the Past on the Web, University of Pennsylvania Press, Philadelphia, 
https://chnm.gmu.edu/digitalhistory/ [Consulta: 05/07/2021].

Cohen, D.J.; Frisch, M.; Gallagher, P.; Mintz, S.; Sword, K.; Taylor, A.; Thomas, W. G.; TURKEL, W. J. (2008): «Interchange: The promise of digital history», The Journal of American History, 95 (2): 452-491. https:/ / doi.org/10.2307/25095630

Dacos, M. (2011): «Manifiesto por unas Humanidades Digitales», Revista hipótesis https:/ / tcp.hypotheses.org/487 [Consulta: 05/07/2021].

Da Silva Caetano, W.; Zarate Sanavria, C. (2021): «The Potential of a Didactic Sequence Using Web 2.0 for Teaching History», Sisyphus: Journal of Education, 1: 41-60. https:// doi.org/10.25749/sis.23284

GALLINI, S.; NoIRET, S. (2011): «La historia digital en la era del Web 2.0. Introducción al dossier Historia digital», Historia Crítica, 43: 16-37.

Gijón Mendigutia, M. (2008): «Los "nuevos historiadores" israelíes: mitos fundacionales y desmitificación", Revista de Estudios Internacionales Mediterráneos (REIM), 1 (5): 27-41.

Haračić, Š. (2012): «Memoricide: A Punishable Behavior?», en P. Davor, P. VJeran y R. VišESLAV (eds.), Confronting the Past: European Experiences, Political Science Research Centre, Zagreb.

HoCKEY, S. (2004): «The History of Humanities Computing», en Susan Schreibman, Ray Siemens y John Unsworth (eds.), A Companion to Digital Humanities, Blackwell, Oxford.

KLEIN, S. (2014): «iNakba: New interactive App documents destroyed Palestinian villages», Haaretz, 5 de mayo. https://www.haaretz.com/new-interactiveapp-documents-nakba-1.5247297 [Consulta: 05/07/2021].

Masalha, N. (2003): Políticas de la Negación: Israel y los refugiados palestinos, Bellaterra, Barcelona.

MASAlHA, N. (2012): Nakba. Limpieza étnica, lucha por la historia, Bellaterra, Barcelona.

Melo Florez, J. (2011): «Historia digital: la memoria en el archivo infinito», Historia Crítica, 43: 82-103. https://doi.org/10.7440/histcrit43.2011.06

MorocutTI, P. (2013): «Education and history for reconciliation in Palestine/Israel. The case of Zochrot», Revista de Paz y Conflictos, 6: 152-171.

Musin, N.; Fisher, E. (2021): «Layers as epistemic and political devices in mobile locative media; the case of iNakba in Israel/Palestine», Continuum: Journal of Media \& Cultural Studies. https:/ / doi.org/10.1080/10304312.2021.1879019

NOIRET, S. (2018): «Trabajar con el pasado en internet: la historia pública digital y las narraciones de las redes sociales», Ayer, 110: 111-140.

PAPpé, I. (2006): La limpieza étnica de Palestina, Grupo Planeta, Barcelona.

PAPpÉ, I. (2020): «An indicative archive: salvaging Nakba documents», Journal of Palestine Studies, 49 (3): 22-40.

Pons, A. (2011): " "Guardar como". La historia y las fuentes digitales», Historia Crítica, 43: 38-61.

Pons, A. (2013): El desorden digital. Guía para historiadoresy humanistas, Siglo XXI, Madrid.

Pons, A. (2018): «El pasado fue analógico, el futuro es digital. Nuevas formas de escritura histórica», Ayer, 110: 19-50.

Pons, A.; EIROA, M. (2018): «Introducción», Ayer, 110: 13-18.

Ramos Tolosa, J. (2015): « ¿No hay eco en el eco? El memoricidio de la Nakba y sus resistencias», Revista de Estudios Internacionales Mediterráneos (REIM), 18 (1): 164-186. https:/ / roderic.uv.es/handle/10550/50507

RAmos TolosA, J. (2020): «La historiografía revisionista israelí: terremoto, giro y 
declive», Revista de Paz y Conflictos, 13 (2): 53-78.

Rojas Castro, A. (2013a): «Las Humanidades Digitales: principios, valores y prácticas», Janus: estudios sobre el Siglo de Oro, 2: 74-99.

Rojas CAstro, A. (2013b): «El mapa y el territorio. Una aproximación históricobibliográfica a la emergencia de las Humanidades Digitales en España», Caracteres. Estudios culturales y críticos de la esfera digital, 2: 10-53.

SELA, R. (2017): «The Genealogy of Colonial Plunder and Erasure - Israel's Control over Palestinian Archives», Social Semiotics, 6 (1): 360-375. http:/ / dx.doi.org/ 10.1080/10350330.2017.1291140

Schreibman, S.; Siemens, R.; Unsworth, J. (2008): A Companion to Digital Humanities, Blackwell, Oxford.

Sleiman, H.; Chebaro, K. (2018): «Narrating Palestine: The Palestinian Oral History Archive Project», Journal of Palestine Studies, 47 (2): 63-76.

SPENCE, P. (2014): «La investigación humanística en la era digital: mundo académico y nuevos públicos», Janus Digital, Annex 2: 117-131.

Svensson, P (2010): «The landscape of Digital Humanities», Digital Humanities, 4 (1).

Ter BraAke, S.; FoKkens, A.; Ockeloen, N.; VAn Son, C. (2016): «Digital History: Towards New Methodologies», en B. Bozic, G. Mendel-Gleason, C. Debruyne y D. O'Sullivan (eds.), Computational History and Data-Driven Humanities, CHDDH 2016, Springer, Cham: 23-32. https://doi.org/10.1007/978-3-31946224-0_3

Toscano, M.; Rabadán, A.; Ros, S.; GonzÁlez-Blanco, E. (2020): «Digital humanities in Spain: Historical perspective and current scenario». Profesional de la información, 29 (6), e290601. https:/ / doi.org/10.3145/epi.2020.nov.01

Zоснгот (2020): Zochrot Annual Report 2019. https://zochrot.org/uploads/ uploads/ 2d2a0576b8db33ef2523c21f62adbd50.pdf [Consulta: 05/07/2021]. 